Fernando F. Fachini

fachini@lcp.inpe.br Inst Nac Pesquisas Espaciais - INPE 12630-000 Cachoeira Pta,.SP. Brazil

\section{Effects of the Initial Droplet Temperature on the Vaporization Process at High Pressure}

The aim of this study is to determine conditions for the quasi-steady regime (QSR) of vaporization of Methanol droplets in high pressure Nitrogen. Under the quasi-steady regime, the square of the droplet radius decreases linearly with time (QSR), a simple result that can be easily implemented in numerical codes. Nevertheless, the vaporization rate cannot be described by a simple expression. The vaporization rate depends initially on the quantity of gas dissolved inside the droplet, on the liquid phase expansion and on the mass loss to the gas phase which is controlled by the initial conditions of the droplet. After this initial period and the droplet heating period, when the heat flux from the gas phase is almost completely employed to vaporize the droplet, the vaporization rate can be expressed approximately by a quasi-steady model. As shown by the numerical results, the main unsteadiness source for the droplet vaporization at high pressure is the droplet heating.

Keywords: droplet vaporization, critical condition

\section{Introduction}

Spray combustion studies have to simultaneously treat processes in several spatial scales. The spray spatial scale is specified by the boundaries of the combustion chamber, and the vaporization scale is characterised by the droplet sizes, therefore, the difference in these scales is of several orders of magnitude. There are many other scales present in this kind of problem, but they are not the focus of this work. Due to the high cost of numerical simulation of spray problems with multiple scales, analytical models are developed for small scale processes to be employed in simulation codes. This work addresses the vaporization problem at high pressure in order to determine the droplet initial conditions leading to a simplified model that can be easily employed in those codes.

At low pressure, the thermal inertia of a region close to the droplet in the gas phase is much smaller than that of the liquid phase (QSR). Approximately, the difference between them is given by the ratio of the ambient gas density to the liquid density, $\varepsilon=\rho_{\infty} / \rho_{\mathrm{l}}$. For normal ambient pressure, the condition $\varepsilon \sim 10^{-3}$ is satisfied and thereby the gas phase behaves quasi-steadily in terms of any characteristic time scale of the liquid phase, i.e. droplet heating period or droplet vaporization. This behaviour leads to the well known linear decrease of the square of the droplet radius with time (Godsave, 1953; Spalding, 1953). For a larger region, with radius about $\varepsilon^{-1 / 2}$ times the droplet radius in the gas phase, counting from the droplet surface, the thermal inertia becomes comparable to that of the liquid phase. Under this condition, the transient processes of accumulation of mass and energy in this broad region have a contribution on the liquid phase processes only of order of magnitude about $\varepsilon^{1 / 2} \sim 1 / 30$ (Crespo and Liñán, 1975; Fachini et al., 1999).

There are some situations in which the representative ambient conditions lead to $\varepsilon \sim 1$ (Givler and Abraham, 1996). This condition is found inside the high-pressure combustion chambers of Diesel engines, turbines and rockets. Furthermore, the conditions can attain several times the thermodynamic critical conditions of the liquid. The gas phase close to the droplet looses its quasi-steady behaviour. The transient processes of mass and energy accumulation in the gas phase close to the droplet have the same importance as the diffusive

Paper accepted May, 2006. Technical Editor: Paulo E. Miyagi. and convective processes on the vaporization rate control. Also, in the high pressure condition, the amount of gases dissolved in the liquid phase increases up to values of the same order of liquid concentrations. The properties of the gas-liquid mixture change drastically from those of the pure liquid.

Despite the transient behaviour of the gas phase, the behaviour of the droplet radius squared is still linear with time under certain conditions. The tendency of the square of the droplet radius is reproduced by imposing the quasi-steady regime for the gas phase. However, the results using this model do not agree, as well as the results obtained by including the gas phase transient regime, with those found experimentally (Zhu et al., 2001). Therefore, the "quasisteady" results obtained from the transient model are a characteristic of the set of transient processes. The droplet initial conditions addressed in this analysis are those that lead to the "quasi-steady" regime for the droplet radius taking into account the transient behaviour of the gas phase.

Moreover, there are other processes, negligible at low pressure, that have a strong influence on the vaporization at high pressure conditions. The ideal gas hypothesis is not justified and, thereby, a real gas equation, such as the cubic equation of state can be used to describe the non-ideal gas behaviour (Zhu and Aggarwal, 2000). The closer the conditions are from the thermodynamic critical conditions, the closer the latent heat of vaporization and the surface tension are from zero. At first glance, the reduction of the latent heat of vaporization would lead to an increase of vaporization rate in the sprays, as shown by the low pressure quasi-steady model (Godsave, 1953; Spalding, 1953), but in reality it leads to a decrease (Lazar and Faeth, 1972). The reduction of the surface tension and the impact of the liquid with high density ambient gas cause the atomization of the liquid in extremely small droplets. Under this condition, the liquid-gas system behaves in a homogeneous regime and the high concentration of the vapor in the environment around the droplets reduces the vaporization rate (Newman and Brzustowski, 1971).

Numerical and experimental results (Canada and Faeth, 1974; Okai et al., 2000) reveal that, at the end of the droplet lifetime, the square droplet radius decreases linearly with time, a consequence of the quasi-steady behaviour of the liquid phase and of the gas phase around the droplet. These results have motivated this work which defined the initial conditions for the droplet that lead to a quasisteady behaviour for the vaporization rate as well as to the d-square 
law suitable for part of the droplet lifetime. A Methanol droplet in high pressure Nitrogen is considered in the analysis.

\section{Nomenclature}

$\bar{a}=$ droplet radius, $m$

$a=$ non-dimensional droplet radius

$\dot{a}=$ time derivative of droplet radius

$\mathrm{a}=$ parameter eq. of state (van der Waals)

$\mathrm{b}=$ parameter eq. of state (van der Waals)

$A=$ parameter eq. of state (Peng-Robinson)

$B=$ parameter eq. of state (Peng-Robinson)

$c_{p}=$ specific heat at constant pressure, $\mathrm{J} / \mathrm{kg} . \mathrm{K}$

$C_{p}=$ specific heat at constant pressure, J/mol.K

$D=$ diffusion coefficient, $m / s$

$f=$ fugacity, bar

$g=\rho / \rho_{c F}$

$h=$ enthalpy, $\mathrm{J} / \mathrm{kg}$

$k=$ thermal conductivity, $W /(m . K)$

$k_{Y}=$ non-dimensional diffusion coefficient

$k_{\theta}=$ non-dimensional thermal conductivity

$K_{i j}=$ Peng-Robinson parameter

$L=$ latent heat of vaporization, $\mathrm{J} / \mathrm{kg}$

Le $=$ Lewis number, $\lambda_{c F} / D\left(T_{c F}\right)$

$L_{\theta}=h c /\left(c_{p c F} T_{c F}\right)$

$\dot{m}=$ vaporization rate, $\mathrm{kg} / \mathrm{s}$

$M=$ molecular weight

$N=$ number of species

$P=$ pressure, bar

$\bar{r}=$ radial coordinate, $m$

$r=$ non-dimensional radial coordinate, $\bar{r} / \bar{a}$

$R=$ universal gas constant, J/mol.K

$t=$ time, $s$

$T=$ absolute temperature, $K$

$u=$ non-dimensional radial velocity, $U \bar{a}_{0} / \lambda_{c F}$

$U=$ radial velocity, $\mathrm{m} / \mathrm{s}$

$X=$ mole fraction

$Y=$ mass fraction

$z=$ compressibility factor

\section{Greek Symbols}

$\beta=$ vaporization constant

$\Delta C_{p}=$ real gas correction for the $\mathrm{C}_{\mathrm{p}}$

$\varepsilon=\rho_{\infty} / \rho_{l}$

$\Gamma=210\left(T_{c} M^{4} / P_{c}^{4}\right)^{1 / 6}$

$\lambda=$ thermal diffusivity, $\mathrm{m}^{2} / \mathrm{s}$

$\omega=$ Pitzer acentric factor

$\rho=$ density, $\mathrm{kg} / \mathrm{m}^{3}$

$\tau=$ non-dimensional time, $t \lambda_{c F} / \bar{a}_{0}^{2}$

$\theta=T / T_{\infty}$

\section{Subscripts}

0 initial condition

$\infty$ ambient condition

c critical condition

eq equilibrium condition

$i$ i species

$F$ droplet-forming substance

$g$ ambient gas

j j species

$l$ liquid condition

$r$ reference condition $v$ vapor condition

\section{Superscripts}

\author{
$L$ liquid condition \\ sat saturation condition \\ $V$ vapor condition
}

\section{Equation of State and Equilibrium Conditions}

Studies were performed to examine the effects of the equation of state on the vaporization and combustion of droplets at high pressure (Zhu and Aggarwal, 2000). These studies pointed out that the Peng-Robinson's equation of state as being the better equation of state for predicting the experimental data over a wide range of pressure. Thereby, it has been employed in droplet problems at high pressure.

$\mathrm{n}$ terms of the compressibility factor $\mathrm{z}$, the Peng-Robinson's equation of state can be expressed (Ohe, 1990) as

$$
z^{3}-(1-B) z^{2}+\left(A-3 B^{2}-2 B\right) z-\left(A B-B^{2}-B^{3}\right)=0
$$

in which

$$
A=\frac{\mathrm{a} P}{R^{2} T^{2}}, \quad B=\frac{b P}{R T}, \quad z=\frac{P}{R_{g} \rho T},
$$

$R_{g}=R / M$ is the gas constant, $R$ is the universal gas constant and $M$ represents the molecular weight. $\mathrm{P}, \mathrm{T}$ and $\rho$ represent the pressure, the temperature and the density, respectively. The parameters a and $b$, which have the same meaning of those of the van derWaals equation of state, represent the binary mixture of gas and are given by the following mixing rule

$$
\mathrm{a}=\sum_{i=1}^{N} \sum_{j=1}^{N} X_{i} X_{j} \mathrm{a}_{i j}, \quad b=\sum_{i=1}^{N} X_{i} b_{i},
$$

in which $X_{i}$ denotes the molar fraction of species $i$. The value of $\mathrm{a}_{i j}$ is defined as.

$$
\mathrm{a}_{i j}=\left(1-K_{i j}\right)\left(\mathrm{a}_{i} \mathrm{a}_{j}\right)^{1 / 2} .
$$

The parameter $K_{i j}$, known as Peng-Robinson's parameter, is evaluated experimentally. For a binary mixture of gases, the value of $K_{i j}$ is presented elsewhere (Ohe, 1990). $\mathrm{a}_{i}$ and $\mathrm{b}_{i}$ are the values of $\mathrm{a}(\mathrm{T})$ and $\mathrm{b}$ for species $\mathrm{i}$,

$$
\begin{aligned}
\mathrm{a}_{i}= & 0.45724\left(R^{2} T_{c}^{2} / P_{c}\right)[1+(0.37464+ \\
& \left.\left.1.54226 \omega-0.26992 \omega^{2}\right)\left(1-T_{r}^{1 / 2}\right)\right]^{2}
\end{aligned}
$$

and

$$
b_{i}=0.07780\left(R T_{c} / P_{c}\right)
$$

in which $T_{r}=T / T_{c}$ is the reduced temperature and the parameter $\omega$, named as the Pitzer acentric factor and defined by $\omega=-\log _{10} P_{r}^{s a t}-1$ at $T_{r}=0.7$. The subscript c identifies the critical conditions.

Since the molecular characteristic time is much smaller than any characteristic time related to the vaporization process, the equilibrium hypothesis at the droplet surface is well justified. 
Thereby, the concentrations at the liquid and vapor sides of the liquid-gas interface depend only on the temperature and pressure. The equilibrium regime is marked by the equality of temperature, pressure and chemical potential at both sides of the interface. For a general system, the equilibrium regime is characterised by the equality of fugacity $f_{i}$ for each species $i$, instead of the chemical potential (Prausnitz, 1969). By making use of the Gibbs-Duhem's equation (Prausnitz, 1969) and of the Peng-Ronbinson's equation of state, the fugacity of species $i$ is evaluated by the expression

$$
\begin{aligned}
& \ln \frac{f_{i}}{X_{i} P}=\frac{b_{i}}{b}(z-1)-\ln (z-B)-\frac{A}{2 \sqrt{2} B} \times \\
& \left(\frac{2}{\mathrm{a}} \sum_{j=1}^{N} X_{j} \mathrm{a}_{i j}-\frac{b_{i}}{b}\right) \ln \left(\frac{z+2.414 B}{z-0.414 B}\right) .
\end{aligned}
$$

The molar fraction of species $i, X_{i}$, is given in terms of the mass fraction $Y_{i}=\rho_{i} / \rho$ by $X_{i}=\left(Y_{i} / M_{i}\right) /\left(\sum_{i} Y_{i} / M_{i}\right)$, in which $M_{i}$ represents the molecular weight of species $i$.

\section{Thermodynamic and Transport Coefficients}

At high pressure condition, the thermodynamic and the transport coefficients have a large difference from those calculated through the ideal gas hypothesis. By considering corrections as a function of the reduced temperature and pressure in the low pressure models, expressions for the real gas specific heat and for the thermal conductivity are determined as a function not only on the temperature, but also on the pressure.

The difference between the real-gas specific heat $C_{p}$ and the ideal-gas specific heat $\mathrm{C}_{\mathrm{p}}{ }^{0}$ can be expressed in terms of

$$
C_{p}-C_{p}^{0}=\left(\Delta C_{p}\right)^{(0)}+\omega\left(\Delta C_{p}\right)^{(1)},
$$

$\omega$ is the same acentric factor. The value of the corrections $\left(\Delta \mathrm{C}_{\mathrm{p}}\right)^{(0)}$ and $\left(\Delta \mathrm{C}_{\mathrm{p}}\right)^{(1)}$ are presented in elsewhere (Reid et al., 1986).

The thermal conductivity $\mathrm{k}$ for real gases can be approximately expressed (Reid et al., 1986) as

$$
\left(k-k_{0}\right) \Gamma z_{c}^{5}=1.14 \times 10^{-2}\left[\exp \left(0.67 \rho_{r}\right)-1.069\right]
$$

in which $z_{c}$ is the compressibility factor at critical condition, $\Gamma=210\left(T_{c} M^{4} / P_{c}^{4}\right)^{1 / 6}$ has the reciprocal thermal conductivity units and $k_{0}$ is the thermal conductivity for low pressure condition. The above expression is valid for $0.5<\rho_{r}<2.0$. The latent heat of vaporization for each species is estimated by the difference of enthalpy at both sides of the liquid-gas interface (Prausnitz, 1969)

$$
L_{i}=h_{i}^{L}-h_{i}^{V}=R_{i} T^{2} \frac{\partial}{\partial T}\left(\ln f_{i}^{V}-\ln f_{i}^{L}\right)
$$

The binary-diffusion coefficient $D$ for high pressures, adopted in this work, follows the Takahashi's model (Takahashi, 1974),

$$
D P=(D P)_{T r \rightarrow \infty}\left(1-\bar{A} T_{r}^{-\bar{B}}\right)\left(1-\bar{C} T_{r}^{-\bar{E}}\right)
$$

in which $\bar{A}, \bar{B}, \bar{C}$ and $\bar{E}$ are functions of the reduced pressure and the value for $(D P)_{T r \rightarrow \infty}$ is close to one.

J. of the Braz. Soc. of Mech. Sci. \& Eng.

\section{Mathematical Formulation}

The present model, describing the droplet vaporization at high pressure, neglects the effects of gravitational and dissipative forces and the heat transfer produced by mass diffusion. Moreover, it is presumed that the droplet and the gas phase around the droplet present spherical symmetry during the lifetime of the droplet, $\bar{a}(t)$ is the droplet radius at time $t$. This problem is marked by the fact that the gas-liquid interface is a free boundary, the eigenvalue of the problem, that is determined when an extra boundary condition is imposed. n computational terms, it is more convenient, through a spatial transformation $r[\equiv \bar{r} / \bar{a}(t)]$, to fix the free frontier than to change the grid of each time step (Crank, 1987). The eigenvalue disappears from the boundary condition, but it appears in the conservation equations.

Under these conditions, the non-dimensional conservation equations (mass, species and energy) present the accumulative, convective and diffusive terms,

$$
\begin{aligned}
& \frac{\partial}{\partial \tau}\left(a^{3} r^{2} g\right)+\frac{\partial}{\partial r}\left[a^{2} r^{2} g(u-r \dot{a})\right]=0, \\
& \frac{\partial}{\partial \tau}\left(a^{3} r^{2} g Y_{F}\right)+\frac{\partial}{\partial r}\left[a^{2} r^{2} g(u-r \dot{a}) Y_{F}\right]= \\
& \frac{\partial}{\partial r}\left(\frac{a r^{2} k_{Y}}{L e} \frac{\partial}{\partial r} Y_{F}\right), \\
& \frac{\partial}{\partial \tau}\left(a^{3} r^{2} g h\right)+\frac{\partial}{\partial r}\left[a^{2} r^{2} g(u-r \dot{a}) h\right]= \\
& \frac{\partial}{\partial r}\left(\frac{a r^{2} k_{\theta}}{L_{\theta}} \frac{\partial}{\partial r} \theta\right),
\end{aligned}
$$

in which $a=\bar{a} / \bar{a}_{0}$ is the non-dimensional droplet radius and $\bar{a}_{0}=\bar{a}(0) ; \dot{a}$ represents the derivative of droplet radius with respect to time ( $d a / d \tau$ ). The non-dimensional time $\tau$ is defined by $t \lambda_{c F} / \bar{a}_{0}^{2}$ (the subscript $F$ denotes the substance from the droplet). $\lambda$, defined by $k /\left(\rho c_{p}\right)$, is the thermal diffusivity. The functions $k_{Y}=D_{F} / D_{c F}$ and $k_{\theta}=k / k_{c}$ retain the dependence of the diffusion coefficient and the thermal conductivity on temperature and concentration.

The non-dimensional radial fluid velocity $u$ corresponds to $U a_{0} / \lambda_{c F}$, the non-dimensional temperature $\theta$ and the nondimensional density $g$ are determined by $\theta=T / T_{c F}$ and $g=\rho / \rho_{c F}$, respectively. The non-dimensional enthalpy $h$ is given by

$$
h=\left(Y_{F} \int_{298}^{T} c_{p F} d T+Y_{N_{2}} \int_{298}^{T} c_{p N_{2}} d T\right) / h_{c}
$$

where

$$
h_{c}=\int_{298}^{T} c_{p F} d T
$$

The parameter $L_{\theta}$ represents $h_{c} /\left(c_{p c F} T_{c F}\right)$ and $L e$ is the Lewis number.

The conservation equations (7) to (9) and the equation of state, Eq. (1), form a closed set of equations, to which is necessary to specify initial and boundary conditions. From the defined problem, 
values for density, velocity, temperature and concentrations are determined inside and outside the droplet

The initial conditions, say at $\tau=0$, are: inside the $\operatorname{droplet}(0 \leq r \leq 1)$ the temperature is uniform and equal to $\theta_{l}=T_{l} / T_{c F}$ and outside the droplet $(1 \leq r)$ the temperature $\theta_{g}=T_{g} / T_{c F}$ is also uniform, but the value is different from that inside the droplet. The pressure is kept constant, $p=P_{\infty} / P_{c F}$, thereby, the initial profile of the density inside the droplet is $g_{l}=\rho_{l} / \rho_{c F}(0 \leq r \leq 1)$ and outside the droplet is $g_{g}=\rho_{g} / \rho_{c F}(1 \leq r)$. Consequently, the velocity is zero everywhere, $\mathrm{u}=0(\forall r)$. By presuming that liquid is in equilibrium with gas for the initial conditions, the portion of liquid inside the droplet is $Y_{F}=Y_{F_{e q}}\left(T_{l}, P\right) \quad(0 \leq r \leq 1)$. The equilibrium assumption between the droplet substance and the Nitrogen is not unrealistic. $t$ represents the conditions reached by the droplet substance and the Nitrogen, just after the atomization but before starting the vaporization, when the relative velocity between the liquid and gas phases is not negligible, this situation induces recirculation inside the droplet that imposes $Y_{F}=Y_{F_{e q}}\left(r=1^{-} ; T_{l}, P\right)$ everywhere in the droplet.

The boundary conditions satisfied by equations (7) to (9) are specified at the droplet center, at the droplet surface and in the ambient atmosphere. At the center, there is no flux. At the droplet surface, mass, momentum and energy are conserved and the liquid and gas are in equilibrium. The ambient conditions are imposed in a numerical finite domain, which is large enough to represent well the ambient atmosphere. Mathematically, these conditions are expressed by

$$
\begin{aligned}
& u=\frac{\partial g}{\partial r}=\frac{\partial Y_{F}}{\partial r}=\frac{\partial \theta}{\theta r}=0 \text { at } r=0 \\
& {[g(u-\dot{a})]_{r=1^{-}}=[g(u-\dot{a})]_{r=1^{+}}} \\
& {\left[g(u-\dot{a}) Y_{F}-\frac{k_{Y}}{L e} \frac{\partial Y_{F}}{\partial r}\right]_{r=1^{-}}=} \\
& {\left[g(u-\dot{a}) Y_{F}-\frac{k_{Y}}{L e} \frac{\partial Y_{F}}{\partial r}\right]_{r=1^{+}}} \\
& {\left[g(u-\dot{a}) h-\frac{k_{\theta}}{L_{\theta}} \frac{\partial \theta}{\partial r}\right]_{r=1^{-}}=} \\
& {\left[g(u-\dot{a}) h-\frac{k_{\theta}}{L_{\theta}} \frac{\partial \theta}{\partial r}\right]_{r=1^{+}}} \\
& \theta\left(r=1^{-}\right)=\theta\left(r=1^{+}\right), \\
& \left.f_{F}^{L}\right|_{r=1}=\left.f_{F}^{V}\right|_{r=1} \\
& u=g-g_{\infty}=Y_{F}=\theta-\theta_{\infty}=0 \quad r \rightarrow \infty
\end{aligned}
$$

The extra condition, from which the droplet radius $a(\tau)$ is determined, is the equilibrium condition for the ambient gas at the droplet surface. By writing it in terms of the mass fraction of the substance that composes the droplet $Y_{N_{2}}=1-Y_{F}$, it follows that

$$
\left.f_{g}^{L}\right|_{r=1}=\left.f_{g}^{V}\right|_{r=1}
$$

Note that the integration of the mass conservation equation (7) from the center to the surface of the droplet,

$$
\dot{m}=-\frac{d}{d r}\left(a^{3} \int_{0}^{1} r^{2} g d r\right)=\left.a^{2} g(u-\dot{a})\right|_{r=1}
$$

defines the vaporization rate $\dot{m}$.

The system of equations (7) to (9) is integrated numerically satisfying the initial conditions and the boundary conditions expressed by Eqs. (10) to (16). Since the flow is generated only by the thermal expansion (Stefan flow) and the phase change of the substance of the droplet, the velocity is much smaller than that of the sound. Thereby, pressure changes could be neglected. $n$ addition, for the ambient condition close to the substance critical conditions, the contribution of the phase change on the flow is of the same order of that produced by the thermal expansion. Due to the problem characteristic, two simple strategies can be applied to solve: One of them works with mass contained up to a spatial coordinate $r$, thus the independent spatial variable is $\phi$ (material function). Thereby, the new spatial coordinate has the following characteristics: $\partial \phi / \partial r=a^{3} r^{2} g$ and $\partial \phi / \partial \tau=-a^{3} r^{2} g(u-r \dot{a})$. The expressions for $\partial \phi / \partial r$ and $\partial \phi / \partial \tau$ satisfy the mass conservation equation. $\mathrm{n}$ addition, the transformation from $(\tau, r)$ to $(\tau, \phi)$ leads to equations of transient-diffusive type.

The other strategy is to fix the droplet surface with the transformation $r=\bar{r} / \bar{a}$. This procedure was adopted in this analysis because there is an advantage in having the eigenvalue $a$ in the system of equations instead of having it in the boundary conditions, as already mentioned.

The finite difference method was used to the discretization of the equations in a implicit scheme (Oran and Boris, 1987; Fletcher, 1991; Ferziger and Peric, 1999). The system of nonlinear algebraic equations was solved by the Newton-Raphson Method employing the LU decomposition to solve the linear algebraic equations (Press et al., 1988). n each time step, a density profile was guessed and the velocity, mass fraction $Y_{F}$ and temperature $\theta$ were determined by Eqs. (7) to (9). At same time the accuracy of the guessed density profile was verified by the equation of state Eq. (1) and a new guessed density profile was found. This procedure was repeated up to satisfying the established accuracy criteria.

This code was considered validated when the linear decrease behaviour of the droplet radius square at the end of the droplet lifetime was obtained as pointed out by previous analyses (Canada and Faeth, 1974; Okai et al., 2000).

\section{Results and Comments}

The model developed here was applied to the vaporization of Methanol droplets with $T_{c}=512.6 \mathrm{~K}$ and pressure $P_{c}=80$ bar in an atmosphere containing only Nitrogen, at a temperature of $800 \mathrm{~K}$ and at the pressure of 75 bar. The ambient temperature is representative of self-ignition conditions of droplet combustion (Kadota et al., 1998; Ruszalo and Hallett, 1992). The pressure is close to the critical pressure of the Methanol. The states of the gas and of the liquid, which is presumed to be a dense gas, are specified by the Peng-Robinson's equation of state. Also, the liquid-gas interface has been assumed to be in equilibrium immediately after the droplet is formed in the gas phase. This initial condition is justified because the equilibrium is achieved in a characteristic time much shorter than that of the conduction.

The choice of the Methanol as the analized substance is based on the fact that its molecular weight is very close to the molecular weight of the Nitrogen, ambient substance. Thereby, the effects of the number of moles of the substances could be neglected. $\mathrm{n}$ first approximation, the assumption $Y_{i}=X_{i}$ could be used. 
The present results show the vaporization process of a Methanol droplet in a quiescent atmosphere. Three initial temperatures for the droplet were considered: 300,350 and $400 \mathrm{~K}$; or in terms of the nondimensional temperature, $\theta=T / T_{C}: 0.585,0.683$ and 0.780 , respectively.

For the pair Methanol and Nitrogen and under the conditions considered here, the Lewis number Le, is equal to 5.86; its value is in accordance with previous work (Harstad and Bellan, 1999). Also, the parameter $L_{\theta}$ is equal to 5.58 .

$\mathrm{n}$ general, the vaporization process is controlled by the heat flux from the gas phase to the liquid phase. Part of this flux is employed to heat up the droplet and the other part is used to vaporize the droplet. Contrary to the low pressure condition where the droplet heating process occurs almost separately from the vaporization process, for high pressure conditions, the vaporization initiates simultaneously with the heating up after the droplet is formed. The two processes take place together as a consequence of the low latent heat. Also, the droplet evaporation is marked, not only by the gas phase heat flux, but also by the initial energetic state of the droplet.

The set of Figs. 1, 2, 3, and 4 shows the evolution of some parameters of the droplet.

Figure 1 displays the evolution of the temperatures at the center and at the surface of the droplet. To highlight each initial condition, the same line style is used for both temperatures; curves for higher temperature represent the condition at the droplet surface and curves for lower temperature represent the condition at the droplet center. The results show that the droplet heating process occurs during the whole droplet life (Jia and Gogos, 1999), as pointing out the difference between the two temperatures (at center and surface). $\mathrm{n}$ addition, the droplet surface temperature has a fast increase in the first moments of vaporization as a result of the large heat flux from the gas phase to the liquid phase, generated by the difference of the temperatures between the two phases. However, the droplet center temperature takes a certain time to show visually changes. For an initial temperature of the droplet equal to $400 \mathrm{~K}(\theta=0.78)$, it is observed that the temperature at the surface decreases initially, but increases after a short period of time. This cooling process occurs due to the vaporization supported by the initial energetic state of the droplet that imposes a large concentration of Methanol vapor at the surface that, consequently, imposes a large Methanol mass flux to the ambient atmosphere. The cooling process is not significant in the other two cases ( 350 and $300 \mathrm{~K}$ ) because the heat loss driven by the vaporization can be compensated by the heat flux from the gas phase.

The temperature at the surface reaches the same value for all three cases. This is a indication that value of $440.8 \mathrm{~K}(\theta=0.86)$ is the equilibrium temperature for the mixture of Methanol and Nitrogen in an ambient pressure of 75 bar.

The evolution of the center temperature for the case with an initial temperature of $400 \mathrm{~K}$ shows initially a little increase that is not observed in the other cases. Since it was assumed in this model that the liquid phase attained immediately an equilibrium composition of Methanol and Nitrogen, the initial heating of the whole droplet is caused by the Nitrogen dissolved inside the droplet. This is not seen in the other cases because the initial equilibrium composition has a very small concentration of Nitrogen in the liquid phase.

For an initial droplet temperature equal to $300 \mathrm{~K}$, it is shown that the vaporization rate is negative, which means that the gain of mass due to the Nitrogen dissolved in the liquid is larger than the loss of Methanol mass to the atmosphere.

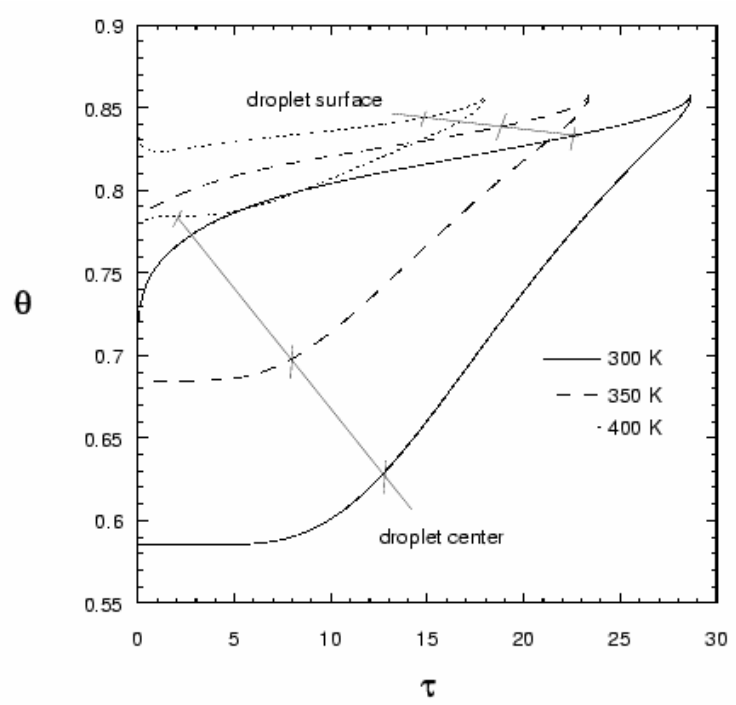

Figure 1. The evolution of the temperature at the center lower curves) and at the surface higher curves) of a Methanol droplet in Nitrogen atmosphere, for three representative initial droplet temperatures. The ambient temperature is $800 \mathrm{~K}$ and the ambient pressure is $75 \mathrm{bar}$.

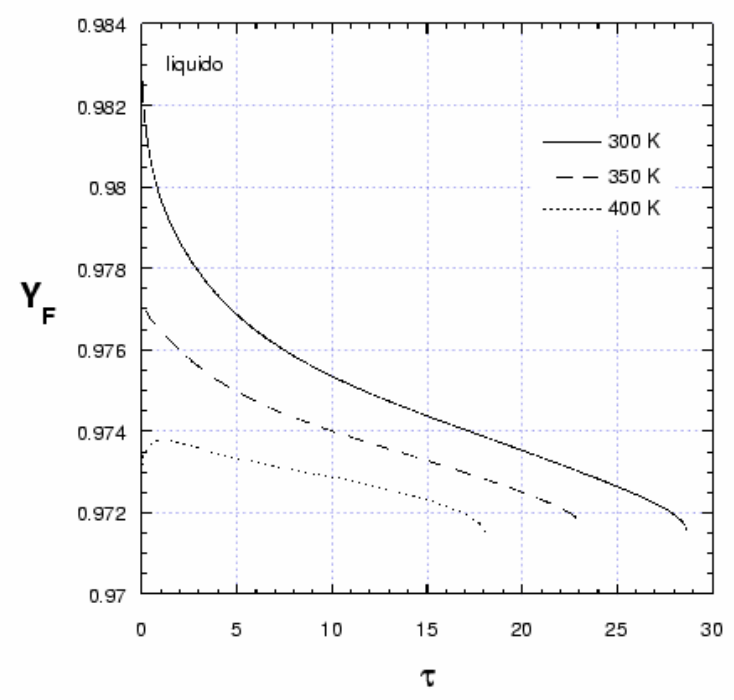

Figure 2. Evolution of Methanol mass fraction in the liquid side of the droplet surface for three representative initial droplet temperatures: 300 , 350 and $400 \mathrm{~K}$. The ambient temperature is $800 \mathrm{~K}$ and the ambient pressure is 75 bar.

The Methanol mass fraction in the liquid and gas sides at the droplet surface is presented in Figs. 2 and 3. The same behaviour observed in the temperature profiles is noted in the concentration profiles. For an initial droplet temperature of $400 \mathrm{~K}$, the initial mass fraction of liquid Methanol is lesser than the other two initial mass fraction, for initial droplet temperatures of 350 and $300 \mathrm{~K}$. However, at the end of lifetime of the droplet, those mass fractions reach the same value, that corresponds to the condition $Y_{F l}=0.9715$. The results for the Methanol mass fraction in the gas phase side at the droplet surface show the influence of the cooling process for an initial droplet temperature of $400 \mathrm{~K}$. 


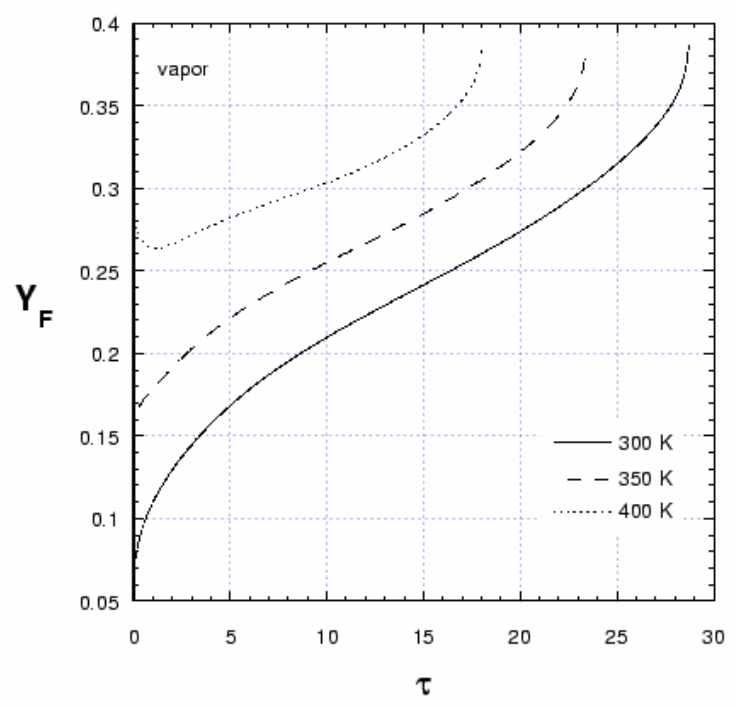

Figure 3. Evolution of the Methanol mass fraction in the gas side of the droplet surface, for three representative initial droplet temperatures: 300 , 350 and $400 \mathrm{~K}$. The ambient temperature is $800 \mathrm{~K}$ and the ambient pressure is 75 bar.

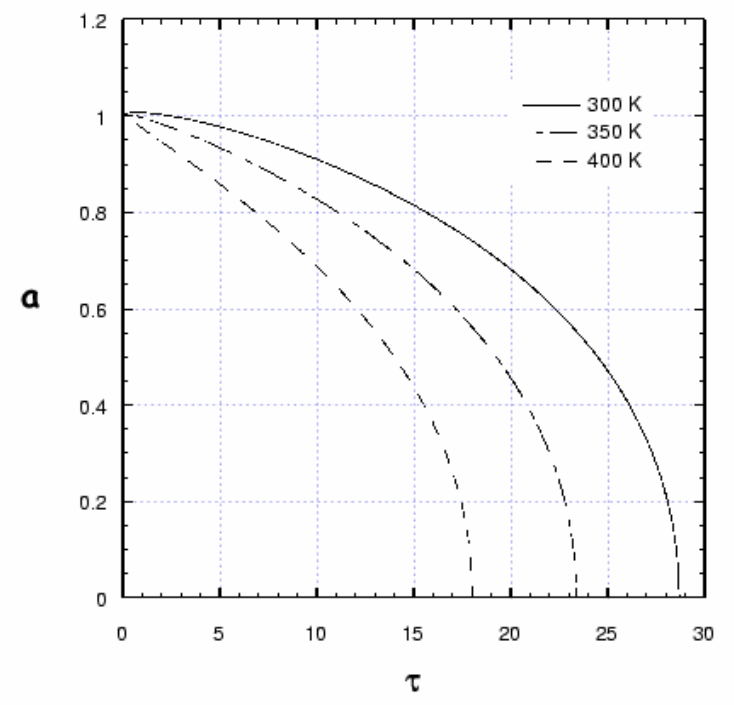

Figure 4. Evolution of the Methanol droplet radius a for three representative initial droplet temperatures: 300,350 and $400 \mathrm{~K}$. The ambient temperature is $800 \mathrm{~K}$ and the ambient pressure is 75 bar.

The Methanol mass fraction decreases initially, but after the cooling process it increases during the lifetime of the droplet. Close to the end of the droplet lifetime, the Methanol concentration in gas phase increases faster. The equilibrium composition for a temperature equal to $440.8 \mathrm{~K}$ and pressure equal to 75 bar does not show $\mathrm{Y}_{\mathrm{F} 1} \sim \mathrm{Y}_{\mathrm{Fv}}$. However, the fast increase of $\mathrm{Y}_{\mathrm{F} 1}$ at the end of the droplet lifetime, indicates that the condition $\mathrm{T}=440.8 \mathrm{~K}, \mathrm{P}=75 \mathrm{bar}$ is very close to the critical condition of the mixture (Ohe, 1990).

As seen in Fig. 4, the evolution of the droplet radius a shows the behaviour $a \sim \tau^{m}$ by the end of the vaporization process. As the droplet lifetime is approached, the derivative of the curve shifts to infinity. From Fig. 5, it is noted that the value of $\mathrm{m}$ is $1 / 2$, the quasisteady result found in low pressure condition. However, for the highest initial droplet temperature, $400 \mathrm{~K}$, the variation of the square droplet radius follows almost exactly the expression $a=1-\beta \tau$
Recalling that for low pressure condition (QSR), the value of $\beta$ is a constant, called vaporization constant, and $\beta$. a is proportional to the vaporization rate, because the density inside the liquid phase is constant. Note that $\beta=-d a^{2} / d \tau$. As the initial droplet temperature is reduced, linear dependence of the square droplet radius on time becomes less representative to the numerical results. $\beta$ is no longer a constant but a time dependent function.

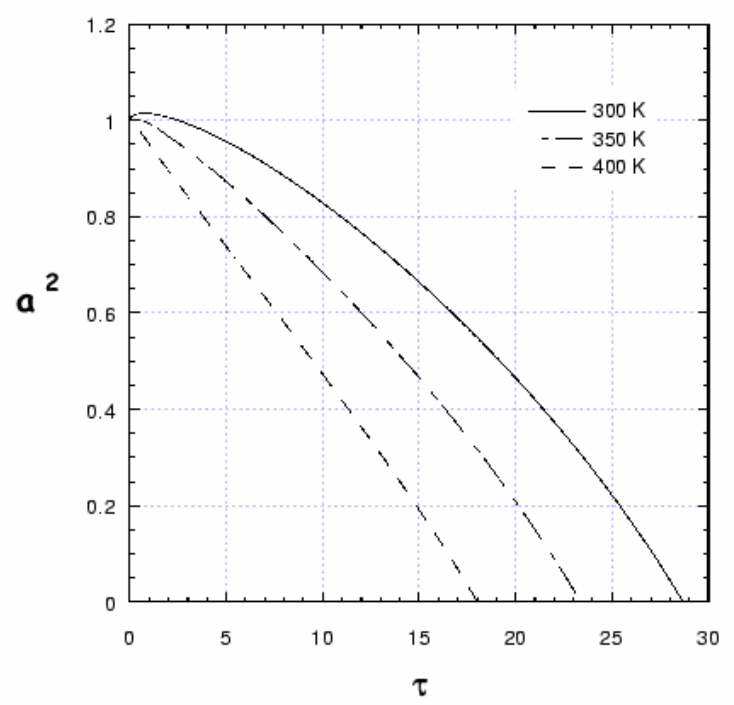

Figure 5. Evolution of the square of the non-dimensional Methanol droplet radius $\mathrm{a}^{2}$, for three initial droplet temperatures: 300,350 and $400 \mathrm{~K}$. The ambient temperature is $800 \mathrm{~K}$ and the ambient pressure is $75 \mathrm{bar}$.

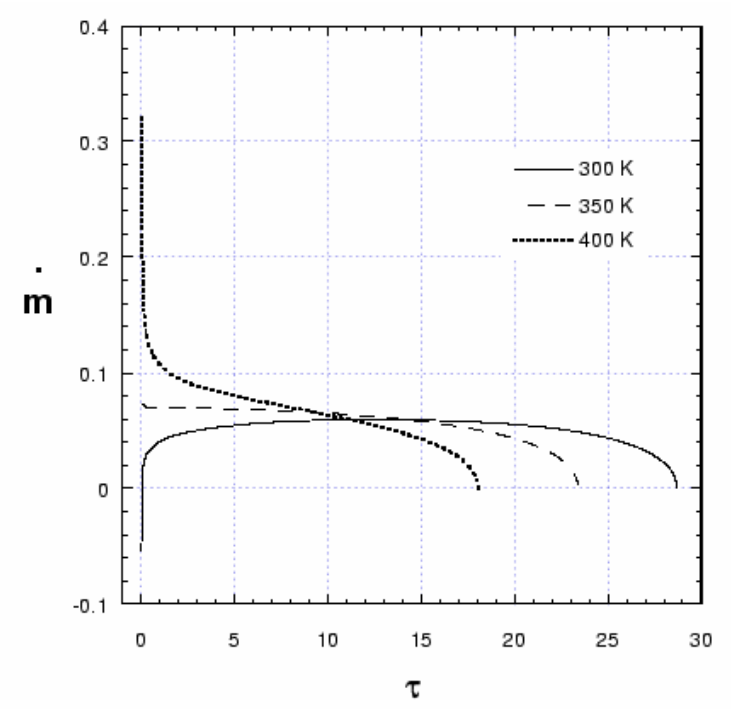

Figure 6. Variation of the non-dimensional vaporization rate $\mathrm{m}^{\circ}$ as a function of time $\tau$, for three representative initial Methanol droplet temperatures: 300,350 and $400 \mathrm{~K}$. The ambient temperature is $800 \mathrm{~K}$ and the ambient pressure is 75 bar.

The vaporization rate $\dot{m}$ for the three initial droplet temperature is presented in Fig. 6. The vaporization rate for the temperature of $400 \mathrm{~K}$ is controlled mainly by the Methanol diffusive flux into the gas phase in the first stages of the vaporization. The mass flux is large and is imposed by the difference of the concentration from the surface, $Y_{F}=0.27$, to the ambient atmosphere, $Y_{F}=0$; as pointing an 
analysis of Eq. (12) with the information $\left.\Delta Y_{F}\right|_{r=1^{-}} \sim 0$ a2nd $\left.\Delta Y_{F}\right|_{r=1^{+}} \sim 0.27$. After this short period, the vaporization rate is driven by the heat flux coming from the gas phase.

The energetic state for the case of the initial droplet temperature of $300 \mathrm{~K}$ is such that the Methanol mass fraction at the vapor side of the liquid-gas interface is not large enough to impose a positive vaporization rate. The conditions lead to a gain of mass of the droplet caused by the gas dissolved in the liquid phase. Thereby, the initial vaporization rate in Fig. 6 for this initial temperature shows a negative value. For the intermediate initial droplet temperature, 350 $\mathrm{K}$, the vaporization rate profile is almost constant up to the half of the droplet lifetime. $\mathrm{n}$ the other half, the vaporization rate is close to that described by the quasi-steady model.

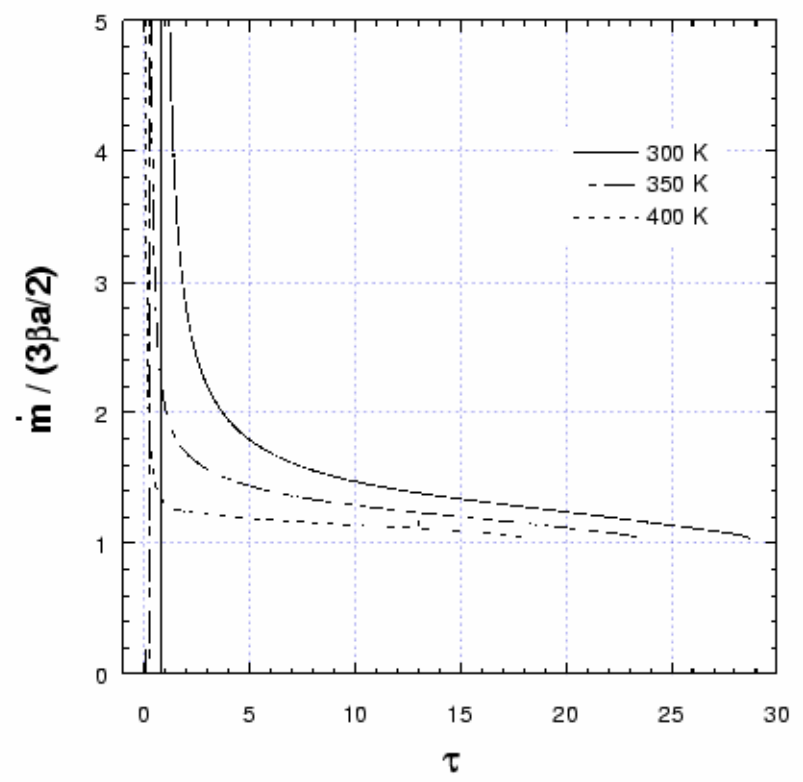

Figure 7. The ratio of non-dimensional vaporization rate $\dot{m}$ to $3 \beta \mathrm{a} / 2$ ) as a function of time $\tau$, for three representative initial droplet temperatures: 300,350 and $400 \mathrm{~K}$. The ambient temperature is $800 \mathrm{~K}$ and the ambient pressure is 75 bar.

Despite the fact that the vaporization is an unsteady process during the whole droplet lifetime, there are situations in which the quasi-steady model is a good approximation to describe the square droplet radius variation, as seen in Fig. 5 for the initial temperature of $400 \mathrm{~K}$, and the vaporization rate, as seen in Fig. 6. Fig. 7 shows the ratio of the nondimensional vaporization rate to $(3 \beta a / 2)$, that would be the vaporization rate in the QSR. The results for the temperature of $400 \mathrm{~K}$ display that the transient regime occurs in the very beginning of the vaporization process, but after that the vaporization rate is very close to that described by the QSR; $\dot{m} /(3 \beta a / 2) \sim 1$. Note that $\beta=-d a^{2} / d \tau$ and $3 \beta a / 2=-d a^{3} / d \tau$, the evaluation of $\beta$ is at the end of the droplet lifetime.

From the results presented here, i.e. the difference of the droplet center and droplet surface temperatures $\left(\theta_{s}-\theta_{l}\right)$, it is observed that the droplet heating is present in the whole droplet lifetime, Thereby, it is the main process responsible by the unsteadiness for the droplet vaporization at high pressure. Therefore, by increasing the initial temperature of the droplet, the problem unsteadiness is reduced and the variation of the square of the droplet radius is approximated by a linear function of time, like that one given by the QSR. Also, the vaporization rate can be represented by the expression of the QSR.
Due to the lack of experimental data on Methanol droplet vaporization at pressures close to the critical condition, the numerical results from this model were not compared to measured results.

\section{Conclusions}

The analysis revealed that, depending on the initial droplet temperature, the behaviour of the droplet radius and the vaporization rate can be described by a quasi-steady model, although the processes in the gas phase is transient during all the droplet lifetime. $t$ seems that the movement of the interface liquid-gas does not cause a strong unsteadiness.

The vaporization process can have two different regimes during the droplet lifetime depending on the initial temperature of the droplet. For the initial temperature far from the critical condition, a unique regime controls the vaporization, i.e. the heat flux from the gas phase to the liquid phase. For the initial temperature close to the critical condition, the initial energetic state of the droplet is able to impose a large mass flux from the liquid phase to the gas phase, independently from the heat flux from the gas phase. During the period of time in which the initial droplet energetic state controls the vaporization, the droplet is cooling. The cooling process takes place in a short period of time because the decrease of the droplet temperature increases the heat flux from the gas phase to the liquid phase. After this short period of time, the heat flux is the process that controls the vaporization.

By playing with these two regimes, changing the initial droplet temperature, it is possible to have an almost constant vaporization rate up to half of the droplet lifetime and after that the vaporization rate follows approximately that one determined by the quasi-steady model.

\section{Acknowledgements}

The research was supported in part by Fundação ao Amparo a Pesquisa do Estado de São Paulo - FAPESP under the Grants 00/08997-4 and 00/08998-0 and by Conselho Nacional de Desenvolvimento Científico e Tecnológico - CNPq under the Grant 302801/03-0.

\section{References}

Canada, G., Faeth, G., 1974. Combustion of liquid fuels in a flowing combustion gas environment at high pressures. Proc. Combust. nstit., Vol. 15, pp. 419-428.

Crank, J., 1987. Free and Moving Boundary Problems. Oxford University Press, Science Oxford Publications, Oxford, UK.

Crespo, A., Liñán, A., 1975. Unsteady effects in droplet evaporation and combustion. Combust. Sci. Techn., Vol.11, pp. 9-18.

Fachini, F. F., Liñán, A., Williams, F. A., 1999. Theory of flame histories in droplet combustion at small stoichiometric fuel-air ratios. A AA Journal, Vol.37, pp. 1426-1435.

Ferziger, J. H., Peric, M., 1999. Computational Methods for Fuid Dynamics. Springer-Verlag, Berlin.

Fletcher, C. A. J., 1991. Computational Techniques for Fuid Dynamics, Vol 1. Springer-Verlag, Berlin.

Givler, S., Abraham, J., 1996. Supercritical droplet vaporization and combustion studies. Prog. Energy Comb. Sc., Vol. 22, pp. 1-28.

Godsave, G., 1953. Studies of the combustion of drops in a fuel spray the burning of single droplets of fuel. Proc. Combust. nstit., Vol.4, pp. 818830 .

Harstad, K., Bellan, J., 1999. A validated all-pressure fluid drop model \& and lewis number effects for a binary mixture. 37th AIAA Aerospace Sciences Meeting and Exhibit, A AA paper 1999-2206, Reno NV, Jan. 10-13.

Jia, H., Gogos, G., 1999. nvestigation of liquid droplet evaporation in subcritical and supercritical gaseous environment. J. Thermophysics Heat Transfer, Vol. 6, pp. 738-745.

Kadota, T., Satoh, K., Segawa, D., Sato, J., Marutani, Y., 1998. Autoignition and combustion of a fuel droplet in supercritical gaseous environments under microgravity. Proc. Combust. nstit., Vol. 27, pp. 25952601 . 
Lazar, R. Faeth, G., 1972. Bipropellant droplet combustion in the vicinity of the critical point. Proc. Combust. nstit., Vol. 13, pp. 801-811.

Newman, J., Brzustowski, T., 1971. Behavior of a liquid jet near the thermodynamic critical region. A AA Journal, Vol. 9, pp. 1595-1602.

Ohe, S., 1990. Vapor-Liquid Equilibrium Data at High Pressure. Kodansha-Elsevier, Tokyo.

Okai, K., Moriue, O., Araki, N., Tsue, M., Kono, M., Sato, J., Dietrich, D., Williams, F., 2000. Pressure effects on combustion of methanol and methanol/dodecanol single droplet and droplet pairs in microgravity. Comb. Flame, Vol. 121, pp. 501-512.

Oran, E. S., Boris, J. P., 1987. Numerical Sumilation of Reactive Flow. Elsevier, New York.

Prausnitz, J. M., 1969. Molecular Thermodynamics of Fluid-Phase Equilibria. Printice-Hall, nc., Englewood Cliffs, N. J.

Press, W. H., Flannery, B. P., Teukolsky, S. A., Vetterling, W. T., 1988. Numerical Recipes. Cambridge University Press, Cambridge.
Reid, R., Prausnitz, J., Poling, B., 1986. The Properties of Gases and Liquids. McGraw-Hill,, Nwe York.

Ruszalo, R., Hallett, W., 1992. A model for the autoignition of single liquid droplets at high pressure. Comb. Sc. Technol., Vol. 86, pp. 183-197.

Spalding, D., 1953. The combustion of liquid fuels. Proc. Combust. nstit., Vol. 4, pp. 847-864.

Takahashi, S., 1974. Preparation of a generalized chart for the diffusion coefficients of gases at high pressures. Journal of Chemical Engineering of Japan, Vol. 7, pp.417-420.

Zhu, G., Aggarwal, S., 2000. Transient supercritical droplet vaporization with emphasis on the effects of equation of state. nt. J. Heat Mass Transfer, Vol.43, pp. 1157-1171.

Zhu, G., Reitz, R., Aggarwal, S., 2001. Gas-phase unsteadiness and its influence on the droplet vaporization in suband super-critical environment. nt. J. Heat Mass Transfer, Vol. 44, pp. 3081-3093. 